\title{
ON THE PROBABILITY OF GENERATING A MINIMAL $d$-GENERATED GROUP
}

\author{
F. DALLA VOLTA, A. LUCCHINI and F. MORINI
}

To Laci Kovács on his 65 th birthday

(Received 9 January 2001; revised 15 June 2001)

\author{
Communicated by R. A. Bryce
}

\begin{abstract}
We consider finite groups with the property that any proper factor can be generated by a smaller number of elements than the group itself. We study some problems related with the probability of generating these groups with a given number of elements.
\end{abstract}

2000 Mathematics subject classification: primary 20B05, $20 \mathrm{P} 05$.

\section{Introduction}

We denote by $\mathscr{L}$ the set of finite groups $L$ with the following properties: $L$ has a unique minimal normal subgroup, say $M$, and if $M$ is abelian then $M$ has a complement in $L$. Let $L_{0}=L / M$ and for any positive integer $t$ define $L_{t}=\left\{\left(l_{1}, \ldots, l_{t}\right) \in L^{t} \mid l_{1} \equiv\right.$ $\left.\cdots \equiv l_{r} \bmod M\right\}$.

Denote by $d(G)$ the minimal number of generators of a finite group $G$; in [2] it is proved that for any nontrivial finite group $G$ there exists $L \in \mathscr{L}$ and a positive integer $t$ such that $L_{t}$ is an epimorphic image of $G$ and $d(G)=d\left(L_{t}\right)>d\left(L_{t-1}\right)$. In particular, if $G$ is a minimal $d$-generated group (meaning by this expression that $d(G)=d$ but $d(G / N)<d$ whenever $N$ is a nontrivial normal subgroup of $G$ ) then $G \cong L_{t}$ for a suitable choice of $L \in \mathscr{L}$ and $t \in \mathbb{N}$. This motivates our interest in the generation properties of groups $L_{t}$; results in this direction can be applied to obtain more general results on the generation of finite groups.

(c) 2001 Australian Mathematical Society 0263-6115/2001 \$A2.00+0.00 
For any finite group $G$, let $\phi_{G}(s)$ denote the number of $s$-bases, that is, ordered $s$ tuples $\left(g_{1}, \ldots, g_{s}\right)$ of elements of $G$ that generate $G$. The number $P_{G}(s)=\phi_{G}(s) /|G|^{s}$ gives the probability that $s$ randomly chosen elements of $G$ generate $G$. Recently Pak [10] introduced the following interesting conjecture: given a real number $\alpha$ with $0<\alpha<1$ there exists an absolute constant $\beta$ such that for any finite group $G$, if $s \geq \beta d(G) \log \log |G|$ then $P_{G}(s) \geq \alpha$.

One of the aims of this paper is to analyse the behaviour of groups $L_{t}$ with respect to this conjecture. We give an evidence for the conjecture proving that if $s$ is large enough with respect to $d\left(L_{t}\right) \log \log \left|L_{t}\right|$ and the probability of generating $L_{0}$ with $s$ element is high, then the probability of generating $L_{t}$ with $s$ elements is also high. To state this result in a more precise way we recall a definition. If $G$ is a finite group and $N$ is a normal subgroup of $G$, let $P_{G, N}(s)=P_{G}(s) / P_{G / N}(s)$. This number is the probability that an $s$-tuple generates $G$, given that it generates $G$ modulo $N$. If $L \in \mathscr{L}$ and $M=\operatorname{soc} L$ then $\operatorname{soc} L_{t} \cong M^{t}$ and $L_{t} / \operatorname{soc} L_{t} \cong L_{0}$. Therefore, $P_{L_{t}}(s)=P_{L_{0}}(s) P_{L_{t}, \text { soc } L_{t}}(s)$. Our main result is the following:

THEOREM 1. Given a real number $\alpha$ with $0<\alpha<1$ there exist two absolute constants $\beta_{1}$ and $\beta_{2}$ such that for any $L \in \mathscr{L}$ and any $t \in \mathbb{N}$

(a) if $\operatorname{soc} L$ is abelian and $s \geq \beta_{1}+d\left(L_{t}\right)$, then $P_{L_{t}, \operatorname{soc} L_{t}}(s) \geq \alpha$;

(b) if $\operatorname{soc} L$ is non abelian and $s \geq \beta_{2} \log (t+1)$, then $P_{L_{t}, \operatorname{soc} L_{t}}(s) \geq \alpha$.

This is a consequence of two more precise results. Let $\mathscr{L}_{\mathrm{ab}}$ be the set of finite groups $L \in \mathscr{L}$ satisfying the property that $\operatorname{soc} L$ is abelian and let $\mathscr{L}_{\text {nonab }}=\mathscr{L} \backslash \mathscr{L}_{\text {ab. }}$.

THEOREM 2. For any $L \in \mathscr{L}_{\mathrm{ab}}$ and any $t, u \in \mathbb{N}$

$$
P_{L_{t}, \operatorname{soc} L_{t}}\left(d\left(L_{t}\right)+u\right) \geq 1-2^{-u} .
$$

THEOREM 3. There exist two positive real numbers $\eta_{1}$ and $\eta_{2}$ such that for any $L \in \mathscr{L}_{\text {nonab }}$ and any $t, u \in \mathbb{N}$, if $P_{L_{0}}(u)>0$ then

$$
P_{L_{t}, \text { soc } L_{i}}(u) \geq 1-\frac{\eta_{1} t^{2}}{2^{\eta_{2} u}}
$$

The second problem that we want to discuss in this paper is the following: suppose $X, Y \in \mathscr{L}$ with $\operatorname{soc} X=\operatorname{soc} Y$ and let $t \in \mathbb{N}$; can we say something about $d\left(Y_{t}\right)$ if we know $d\left(X_{t}\right)$ ? A partial answer follows from [7, Proposition 1]: if $X, Y \in \mathscr{L}_{\text {nonab, }}$, $\operatorname{soc} X=\operatorname{soc} Y$ and $X \leq Y$ then, for any $t \in \mathbb{N}, d\left(Y_{t}\right) \leq \max \left(d\left(Y_{0}\right), d\left(X_{t}\right)+1\right)$. In this paper we prove a more general result.

THEOREM 4. There exists a positive integer $r$ with the following property: for any pair of groups $X, Y \in \mathscr{L}_{\text {nonab }}$ with $\operatorname{soc} X=\operatorname{soc} Y$ and any non negative integer $t$, $d\left(Y_{t}\right) \leq \max \left(d\left(Y_{0}\right), d\left(X_{t}\right)+r\right)$. 
Note that one cannot expect to bound $d\left(Y_{t}\right)$ only as a function of $d\left(X_{t}\right)$ but independently from $d\left(Y_{0}\right)$. As we will show in Section 4 , for any $t, u \in \mathbb{N}$, there exists a pair $X, Y$ of groups in $\mathscr{L}_{\text {nonab }}$ with soc $X=\operatorname{soc} Y, d\left(X_{t}\right)=2, d\left(Y_{t}\right) \geq d\left(Y_{0}\right)=u$. It is also possible to construct examples with $d\left(Y_{t}\right)>\max \left(d\left(Y_{0}\right), d\left(X_{t}\right)\right)$ while we know no example with $d\left(Y_{t}\right)>\max \left(d\left(Y_{0}\right), d\left(X_{t}\right)+1\right)$. Therefore we can conjecture that one can take $r=1$ in Theorem 4 . We prove that this is true asymptotically.

THEOREM 5. There exists a positive real number $\zeta$ with the following property: for any pair of groups $X, Y \in \mathscr{L}_{\text {nonab }}$ with $\operatorname{soc} X=\operatorname{soc} Y$ and any nonnegative integer $t$, if $|\operatorname{soc} X| \geq \zeta$, then $d\left(Y_{t}\right) \leq \max \left(d\left(Y_{0}\right), d\left(X_{t}\right)+1\right)$.

There are no similar results for pairs $X, Y$ of groups in $\mathscr{L}_{\mathrm{ab}}$. In Section 4 , for any positive integers $n, u$, we construct $X, Y \in \mathscr{L}_{\mathrm{ab}}$ with $\operatorname{soc} X=\operatorname{soc} Y, d\left(X_{0}\right)=2$, $d\left(X_{n u}\right)=u+1, d\left(Y_{0}\right)=1, d\left(Y_{n u}\right)=n u+1$.

\section{Preliminary results}

In this section we describe how the number $P_{L_{t}}(s)$ can be computed.

First assume that $L \in \mathscr{L}_{\mathrm{ab}}$. In this case the socle $M$ of $L$ has a complement $H$ in $L$. Of course $H$ is isomorphic to an irreducible subgroup of Aut $M$. Define the numbers $q_{L}, r_{L}, s_{L}$ and $\theta_{L}$ as follows: $q_{L}=\left|\operatorname{End}_{H} M\right|, q_{L}^{r_{L}}=|M|, q_{L}^{s_{L}}=\left|\mathrm{H}^{\prime}(H, M)\right|, \theta_{L}=0$ or 1 according as $M$ is trivial or not. Moreover, let $h_{L, t}=\theta_{L}+\left\lceil\left(t+s_{L}\right) / r_{L}\right\rceil$, where $\lceil x\rceil$ denotes the smallest integer greater or equal $x$. From [5, Lemma 2] it follows:

PROPOSITION 6. If $L \in \mathscr{L}_{\mathrm{ab}}$, then for any $s, t \in \mathbb{N}$

$$
P_{L}(s)=P_{L_{0}}(s) \prod_{0 \leq i \leq t-1}\left(1-q_{L}^{r_{L}\left(\theta_{L}-s\right)+s_{L}+i}\right) .
$$

In particular, $d\left(L_{t}\right)=\max \left(d\left(L_{0}\right), h_{L, t}\right)$.

If $L \in \mathscr{L}_{\text {nonab }}$ and $M=\operatorname{soc} L$, we may identify $L$ with a subgroup of Aut $M$. Let $\gamma_{L}=\left|C_{\mathrm{Aut} M}(L / M)\right|$ and for any $s \in \mathbb{N}$ define $\psi_{L}(s)=\phi_{L}(s) / \gamma_{L} \phi_{L / M}(s)$. The number $\psi_{L}(s)$ plays an important role in the computation of $P_{L_{r}, \text { soc } L_{i}}(s)$. The following result generalises a formula ([6, Proposition 9]) about the probability of generating a direct product of isomorphic non abelian finite simple groups.

Proposition 7. If $L \in \mathscr{L}_{\text {nonab, }}$ then for any $s, t \in \mathbb{N}$ with $s \geq d\left(L_{0}\right)$,

$$
P_{L_{t}}(s)=P_{L_{0}}(s) P_{L, \text { sac } L}(s)^{t} \prod_{1 \leq i \leq t-1}\left(1-\frac{i}{\psi_{L}(s)}\right) .
$$


PROOF. Let $M=\operatorname{soc} L$ and $C_{L}=C_{\mathrm{Aut} M}(L / M)$. As it is proved in [2] the group $L_{t}$ is generated by $s$ elements $g_{1}=\left(x_{11}, \ldots, x_{1 t}\right), \ldots, g_{s}=\left(x_{s 1}, \ldots, x_{s t}\right)$ if and only if:

(a) for $1 \leq i \leq s,\left(x_{1 i}, \ldots, x_{s i}\right)$ is an $s$-basis of $L$;

(b) if $1 \leq i<j \leq t,\left(x_{1 j}, \ldots, x_{s j}\right) \notin \Gamma_{i}=\left(x_{1 i}, \ldots, x_{s i}\right)^{C_{L}}$.

So, to choose $g_{1}, \ldots, g_{s}$ generating $L_{t}$ we first choose an $s$-basis $\left(x_{11}, \ldots, x_{s 1}\right)$ of $L$, and this can be done in exactly $\phi_{L}(s)$ different ways. Let $\Omega_{x_{11} \ldots, x_{s t}}=\left\{\left(y_{1}, \ldots, y_{s}\right) \in\right.$ $L \mid y_{i} \equiv x_{i 1} \bmod M, 1 \leq i \leq s$, and $\left.\left\langle y_{1}, \ldots, y_{s}\right\rangle=L\right\}$. If $i>1,\left(x_{1 i}, \ldots, x_{s i}\right) \in$ $\Omega_{x_{11}, \ldots, x_{s 1}} \backslash\left(\Gamma_{1} \cup \ldots \cup \Gamma_{i-1}\right)$. By a result of Gaschütz [4] $\left|\Omega_{x_{11}, \ldots, x_{s} \mid}\right|=\phi_{L}(s) / \phi_{L / M}(s)$. Moreover, the sets $\Gamma_{i}, 1 \leq i \leq t$, are pairwise disjoint and, being $\left\langle x_{1 i}, \ldots, x_{s i}\right\rangle=L$, it must be $\left|\Gamma_{i}\right|=\left|C_{L}\right|=\gamma_{L}$. Therefore, if $i>1,\left(x_{1 i}, \ldots, x_{s i}\right)$ can be chosen in exactly $\left[\phi_{L}(s) / \phi_{L / M}(s)\right]-(i-1) \gamma_{L}$ different ways. So we have

$$
\begin{aligned}
P_{L_{t}}(s) & =\frac{\phi_{L}(s)}{\left|L_{t}\right|^{s}} \prod_{1 \leq i \leq t-1}\left(\frac{\phi_{L}(s)}{\phi_{L / M}(s)}-i \gamma_{L}\right) \\
& =P_{L}(s) P_{L, \mathrm{soc} L}(-s)^{t-1} \prod_{1 \leq i \leq t-1}\left(1-\frac{i}{\psi_{L}(s)}\right) \\
& =P_{L_{0}}(s) P_{L, \operatorname{soc} L}(s)^{t} \prod_{1 \leq i \leq t-1}\left(1-\frac{i}{\psi_{L}(s)}\right)
\end{aligned}
$$

COROLlaRY 8. Assume $L \in \mathscr{L}_{\text {nonab }}, s \geq \max \left(2, d\left(L_{0}\right)\right) ; d\left(L_{t}\right) \leq s$ if and only if $t \leq \psi_{L}(s)$.

PROof. Suppose $s \geq 2$ and $P_{L_{0}}(s)>0$; by the main theorem in [8] $d(L)=$ $\max \left(2, d\left(L_{0}\right)\right)$, so it follows that $P_{L . s o c}(s)=P_{L}(s) / P_{L_{0}}(s)>0$. Therefore, from Proposition $7, P_{L_{t}}(s)>0$ if and only if $1>i / \psi_{L}(s)$ for $1 \leq i \leq t-1$ and this is equivalent to the condition $\psi_{L}(s) \geq t$.

A bound for $\psi_{L}(s)$ can be deduced from the following result ([9, Corollary 1.2]).

PROPOSITION 9. There exists an absolute constant $\gamma, 0<\gamma<1$, such that for any $L \in \mathscr{L}_{\text {nonab }}$ and any integer $s \geq 2$ we have $\phi_{L}(s) \geq \gamma \phi_{L_{0}}(s)|\operatorname{soc} L|^{s}$.

PROPOSITION 10. Suppose that $L \in \mathscr{L}_{\text {nonab }}$ and that $M=\operatorname{soc} L \cong S^{n}$ with $S$ a non abelian simple group. If $\gamma$ is the constant which appears in the statement of Proposition 9, then for any $s \geq \max \left(2, d\left(L_{0}\right)\right)$ we have

$$
\frac{\gamma|M|^{s-1}}{n \mid \text { Out } S \mid} \leq \psi_{L}(s) \leq|M|^{s-1} \text {. }
$$

Proof. By Proposition 9, $\gamma|M|^{s} \leq \phi_{L}(s) / \phi_{L / M}(s) \leq|M|^{s}$. Moreover, from the proof of [3, Lemma 1], $|M| \leq\left|C_{L}\right| \leq n|S|^{n-1} \mid$ Aut $S \mid$. 


\section{Proof of Theorem 1}

In this section, we deal with the proofs of Theorem 2 and Theorem 3; Theorem 1 follows immediately from these two results.

First, in order to prove Theorem 2, we need the following lemma.

LEMMA 11. For any $L \in \mathscr{L}_{\mathrm{ab}}$ and $t, u \in \mathbb{N}$,

$$
P_{L_{t}}\left(h_{L, t}+u\right) \geq P_{L_{0}}\left(h_{L, t}+u\right)\left(1-q_{L}^{-r_{L} u}\right) .
$$

PROOF. By Proposition 6 and noticing that $r_{L}\left(\theta_{L}-h_{L, t}-u\right)+s_{L} \leq-t-r_{L} u$, if $h_{L, t}+u \geq d\left(L_{0}\right)$ we have

$$
\begin{aligned}
P_{L_{r}, \text { soc } L_{t}}\left(h_{L, t}+u\right) & \geq \prod_{0 \leq i \leq t-1}\left(1-q_{L}^{r_{L}\left(\theta_{L}-h_{L, t}-u\right)+s_{L}+i}\right) \geq \prod_{0 \leq i \leq r-1}\left(1-q_{L}^{-r_{L} u-t+i}\right) \\
& \geq 1-\sum_{0 \leq i \leq t-1} q_{L}^{-r_{L} u-t+i} \geq 1-q_{L}^{-r_{L} u} \sum_{1 \leq j \leq t} q_{L}^{-j} \\
& \geq 1-q_{L}^{-r_{L} u} \sum_{1 \leq j \leq \infty} q_{L}^{-j} \geq 1-q_{L}^{-r_{L} u} .
\end{aligned}
$$

PROOF OF THEOREM 2. It follows immediately from Lemma 11 , since, by Proposition $6, h_{L, t} \leq d\left(L_{t}\right)$.

By [1, Theorem A], if $L \in \mathscr{L}_{\text {ab }}$ then $s_{L}<r_{L}$ and this implies $h_{L, t} \leq t+1$. Therefore from Lemma 11 we also deduce:

CoROllaRY 12. For any $L \in \mathscr{L}_{\mathrm{ab}}$ and $s \in \mathbb{N}, P_{L_{t}}(s) \geq P_{L_{0}}(s)\left(1-2^{-(s-t-1)}\right)$.

Now, we are left with the non abelian case. Again, to prove Theorem 3, we start with two lemmas:

Lemma 13. Suppose that $L \in \mathscr{L}_{\text {nonab }}$ and let $M=\operatorname{soc} L$. There exist two positive constants $\sigma_{1}$ and $\sigma_{2}$ such that for any $u \in \mathbb{N}$ with $u \geq \max \left(2, d\left(L_{0}\right)\right)$,

$$
P_{L, M}(u) \geq 1-\sigma_{1} / e^{\sigma_{2} u} .
$$

ProOF. There exist a positive integer $n$ and a non abelian simple group $S$ such that $M=\operatorname{soc} L \cong S^{n}$. Denote by $S_{1}$ the subset of $S^{n}=M$ consisting of elements $x=$ $\left(1, x_{2}, \ldots, x_{n}\right)$ and let $\phi_{1}: N_{L}\left(S_{1}\right) \rightarrow$ Aut $S$ be the map induced by the conjugation action of $N_{L}\left(S_{1}\right)$ on $S$. Select $g_{1}, \ldots, g_{u}$ in $L$ such that $\left\langle g_{1}, \ldots, g_{u}, M\right\rangle=L$. From [9, Lemma 2.12] it follows:

$$
\frac{\phi_{L}(u)}{\phi_{L / M}(u)} \geq\left|\Omega_{1}\right|-|M|^{3 / 2}-|M|^{u / 2+19 / 20}
$$


where $\Omega_{1}=\left\{\left(m_{1}, \ldots, m_{u}\right) \in M^{u} \mid\left(N_{\left\langle g_{1} m_{1}, \ldots, g_{u} m_{u}\right\rangle}\left(S_{1}\right)\right) \phi_{1} \geq S\right\}$. For any $1 \leq i<j \leq$ $u$, define $\Delta_{i, j}=\left\{(x, y) \in M^{2} \mid\left(N_{\left\langle g_{i} x, g_{j} y\right\rangle}\left(S_{1}\right)\right) \phi_{1} \geq S\right\}$.

We can repeat the arguments used in [9, Lemma 2.10] and prove that $\left|\Delta_{i, j}\right| \geq$ $c_{s}|M|^{2}$, where $c_{s}$ is the positive constant which appears in [9, Proposition 2.7]. We note that $\left(m_{1}, \ldots, m_{u}\right)$ is an element of $\Omega_{1}$ if there exists at least a pair $\left(m_{2 i+1}, m_{2 i+2}\right) \in$ $\Delta_{2 i+1,2 i+2}$, where $0 \leq i \leq[u / 2]-1$.

It follows that

$$
\begin{aligned}
\left|\Omega_{1}\right| & \geq|M|^{u}-\prod_{0 \leq i \leq\{u / 2]-1}\left(|M|^{2}-\left|\Delta_{2 i+1,2 i+2}\right|\right) \\
& \geq|M|^{u}-\prod_{0 \leq i \leq\{u / 2]-1}\left(|M|^{2}-c_{s}|M|^{2}\right) \geq|M|^{u}\left(1-\left(1-c_{s}\right)^{[u / 2]}\right) .
\end{aligned}
$$

So we have

$$
P_{L, M}(u) \geq 1-\left(1-c_{s}\right)^{[u / 2]}-\frac{|M|^{3 / 2}}{|M|^{u}}-\frac{|M|^{19 / 20}}{|M|^{u / 2}} .
$$

By $\left[9\right.$, Proposition 2.7] we derive that $c^{*}=\inf _{s} c_{s}$ is a positive number. Set $\eta=1-c^{*}$. Then we have

$$
\left(1-c_{s}\right)^{[u / 2]} \leq \eta^{[u / 2]} \leq \eta^{u / 2-1} .
$$

Moreover, $|M| \geq 60 \geq e^{4}$ and $u \geq 2$ imply that

$$
|M|^{3 / 2-u} \leq e^{-u / 2}, \quad|M|^{19 / 20-u / 2} \leq e^{19 / 5-2 u} \leq e^{4 / 5-u / 2} .
$$

Set $\sigma_{1}=\eta^{-1}+1+e^{4 / 5}$ and $\sigma_{2}=\min (1 / 2,-\log \eta / 2)$ and conclude

$$
\begin{aligned}
P_{L, M}(u) & \geq 1-\eta^{-1} \eta^{u / 2}-e^{-u / 2}-e^{4 / 5} e^{-u / 2} \\
& \geq 1-\eta^{-1} e^{-u \sigma_{2}}-e^{-u \sigma_{2}}-e^{4 / 5} e^{-u \sigma_{2}} \geq 1-\sigma_{1} e^{-u \sigma_{2}} .
\end{aligned}
$$

LEMMA 14. Suppose that $L \in \mathscr{L}_{\text {nonab }}$ and that $M=\operatorname{soc} L \cong S^{n}$ with $S$ a non abelian simple group. For any $t, u \in \mathbb{N}$ with $u \geq 2$

$$
\prod_{1 \leq i \leq t-1}\left(1-\frac{i}{\psi_{L}(u)}\right) \geq 1-\frac{t^{2}}{\gamma 2^{u-2}},
$$

where $\gamma$ is the constant which appears in the statement of Proposition 9.

ProOF. By Proposition 10 and noticing that $n \mid$ Out $S|\leq| S^{n}|=| M \mid$ we have

$$
\begin{aligned}
\prod_{1 \leq i \leq t-1}\left(1-\frac{i}{\psi_{L}(u)}\right) & \geq 1-\frac{\sum_{1 \leq i \leq t-1} i}{\psi_{L}(u)} \geq 1-\frac{(t-1)^{2}}{\psi_{L}(u)} \\
& \geq 1-\frac{(t-1)^{2} n \mid \text { Out } S \mid}{\gamma|M|^{u-1}} \geq 1-\frac{(t-1)^{2}}{\gamma|M|^{u-2}} \geq 1-\frac{t^{2}}{\gamma 2^{u-2}} .
\end{aligned}
$$


PROOF OF THEOREM 3. It follows immediately from Lemma 13, Lemma 14 and Proposition 7. Precisely, noticing that $\sigma_{2} u \leq u-1$ we have

$$
\begin{gathered}
P_{L . M}(u)^{t} \geq 1-\frac{\sigma_{1} t}{2^{\sigma_{2} u}}, \\
\prod_{1 \leq i \leq t-1}\left(1-\frac{i}{\psi_{L}(u)}\right) \geq 1-\frac{2 \gamma^{-1} t^{2}}{2^{u-1}} \geq 1-\frac{2 \gamma^{-1} t^{2}}{2^{\sigma_{2} u}}
\end{gathered}
$$

and hence

$$
P_{L_{i}, \operatorname{soc} L_{t}}(u) \geq 1-\frac{2 \gamma^{-1} t^{2}+\sigma_{1} t}{2^{\sigma_{2} u}} \geq 1-\frac{\left(\sigma_{1}+2 \gamma^{-1}\right) t^{2}}{2^{\sigma_{2} u}} .
$$

\section{Proof of Theorem 4 and Theorem 5}

In this section we consider two groups $X$ and $Y \in \mathscr{L}_{\text {nonab }}$ such that $\operatorname{soc} X=$ soc $Y$. It seems interesting to compare $d\left(X_{t}\right)$ and $d\left(Y_{t}\right)$. As already observed in the introduction, $X \leq Y$ implies $d\left(Y_{t}\right) \leq \max \left(d\left(Y_{0}\right), d\left(X_{t}\right)+1\right)$ ([7]).

One cannot expect to have $d\left(Y_{t}\right) \leq \max \left(d\left(Y_{0}\right), d\left(X_{t}\right)\right)$ for any pair of groups $X, Y \in \mathscr{L}_{\text {nonab }}$ with soc $X=\operatorname{soc} Y$. For example, let $X=\operatorname{PGL}(2,7), Y=\operatorname{PSL}(2,7)$; it can be computed that $\psi_{X}(2)=69$ and $\psi_{Y}(2)=57$, hence, by Corollary $8, d\left(Y_{58}\right)=$ 3 while $d\left(X_{58}\right)=2$. However, we conjecture that $d\left(Y_{t}\right) \leq \max \left(d\left(Y_{0}\right), d\left(X_{t}\right)+1\right)$. From the proof of Theorem 4 , one can deduce that to prove this conjecture if suffices to show that $\gamma \geq 1 / \sqrt{60}$, where $\gamma$ is the constant which appears in the statement of Proposition 9.

We explicitly observe that, in general, it is not true neither that if $X \geq Y$ then $d\left(X_{t}\right) \geq d\left(Y_{t}\right)$ (see the previous example) nor the converse. If we take $Y=\operatorname{PSU}(3,3)$ and $X=\operatorname{Aut}(Y)$, we obtain $\psi_{Y}(2)=2784$ and $\psi_{X}(2)=2772$, so that $2=d\left(Y_{2773}\right)<$ $d\left(X_{2773}\right)=3$.

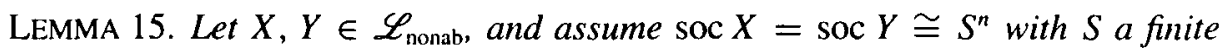
non abelian simple group, and let $r, t \in \mathbb{N}$. If $d\left(Y_{t}\right)>\max \left(d\left(Y_{0}\right), d\left(X_{t}\right)+r\right)$ then $n \mid$ Out $\left.S|/| S\right|^{r n}>\gamma$, where $\gamma$ is the constant which appears in the statement of Proposition 9.

PROOF. Since $\max \left(2, d\left(X_{0}\right)\right) \leq d\left(X_{t}\right)$, by Corollary 8 we have $t \leq \psi_{X}\left(d\left(X_{t}\right)\right)$. On the other hand, again by Corollary $8, d\left(Y_{t}\right)>\max \left(d\left(Y_{0}\right), d\left(X_{t}\right)+r\right)$ implies $t>\psi_{Y}\left(d\left(X_{t}\right)+r\right)$. Using Proposition 10 we deduce

$$
\frac{\gamma|S|^{n\left(d\left(X_{t}\right)+r-1\right)}}{n \mid \text { Out } S \mid} \leq \psi_{Y}\left(d\left(X_{t}\right)+r\right)<t \leq \psi_{X}\left(d\left(X_{t}\right)\right) \leq|S|^{n\left(d\left(X_{t}\right)-1\right)}
$$

which implies $\gamma|S|^{r n}<n \mid$ Out $S \mid$. 
PROOF OF THEOREM 4. Let $r$ be the smallest integer satisfying $r \geq-\log _{60} \gamma+1 / 2$. Suppose by contradiction that there exist $X, Y \in \mathscr{L}_{\text {nonab }}$ and $t \in \mathbb{N}$ such that $\operatorname{soc} X=$ soc $Y$ and $d\left(Y_{t}\right)>\max \left(d\left(Y_{0}\right), d\left(X_{t}\right)+r\right)$. By Lemma 15 , if $\operatorname{soc} X=S^{n}$ with $S$ a non abelian simple group, then $\gamma<n \mid$ Out $\left.S|/| S\right|^{r n}$. On the other hand, $\mid$ Out $S \mid \leq \sqrt{|S|}$ (see [9, Proposition 2.6]) and $|S| \geq 60$, so

$$
\gamma<\frac{n \mid \text { Out } S \mid}{|S|^{r n}} \leq \frac{\mid \text { Out } S \mid}{|S|^{r}} \leq \frac{\sqrt{|S|}}{|S|^{r}} \leq 60^{1 / 2-r}
$$

in contradiction with the choice of $r$.

LEMMA 16. Let $M=S^{n}$ be a direct product of isomorphic non abelian simple groups, then $\lim _{|M| \rightarrow \infty} n \mid$ Out $\left.S|/| S\right|^{n}=0$.

ProOF. By [9, Proposition 2.6]

$$
\frac{n \mid \text { Out } S \mid}{|S|^{n}} \leq \frac{n \sqrt{|S|}}{|S|^{n}} \leq \frac{\sqrt{|S|^{n}}}{|S|^{n}} \leq \frac{1}{\sqrt{|M|}}
$$

PROOF OF THEOREM 5. By Lemma 16 there exists $\zeta$ such that if $M=S^{n}$ is a direct product of isomorphic non abelian simple groups and $|M| \geq \zeta$, then $n \mid$ Out $S|/| M \mid \leq$ $\gamma$. Suppose by contradiction that there exist $X, Y \in \mathscr{L}_{\text {nonab }}$ and $t \in \mathbb{N}$ satisfying $\operatorname{soc} X=\operatorname{soc} Y \cong S^{n},\left|S^{n}\right| \geq \zeta$ and $d\left(Y_{t}\right)>\max \left(d\left(Y_{0}\right), d\left(X_{t}\right)+1\right)$. By Lemma 15 , $\gamma<n \mid$ Out $\left.S|/| S\right|^{n}$, against the choice of $\zeta$.

We have proved that if $X, Y \in \mathscr{L}_{\text {nonab }}$ with $\operatorname{soc} X=\operatorname{soc} Y$, then $d\left(Y_{t}\right)$ can be bounded in terms of $d\left(X_{t}\right)$ and $d\left(Y_{0}\right)$. The next result shows that it is impossible to bound $d\left(Y_{t}\right)$ from the knowledge of $d\left(X_{t}\right)$ but independently from $d\left(Y_{0}\right)$.

PROPOSITION 17. For any $t, u \in \mathbb{N}$, there exists a pair $X, Y$ of groups in $\mathscr{L}_{\text {nonab }}$ with $\operatorname{soc} X=\operatorname{soc} Y, d\left(X_{t}\right)=2, d\left(Y_{t}\right) \geq d\left(Y_{0}\right)=u$.

PROOF. Let $A$ be an elementary abelian 2-group of rank $u$ and let $S$ be a finite non abelian simple group with $|S| \geq\left(2^{u} t / \gamma\right)^{2} ; A$ can be viewed as a regular permutation group of degree $2^{u}$. Consider the wreath products $X=S$ iSym $\left(2^{u}\right)$ and $Y=S \imath A$. Of course $X, Y \in \mathscr{L}_{\text {nonab }}$ and $\operatorname{soc} X=\operatorname{soc} Y=S^{2^{u}}$. By Proposition 10

$$
\psi_{X}(2) \geq \frac{\gamma|S|^{2^{u}}}{2^{u} \mid \text { Out } S \mid} \geq \frac{\gamma|S|^{2^{u}}}{2^{u} \sqrt{|S|}} \geq \frac{\gamma \sqrt{|S|}}{2^{u}} \geq t,
$$

hence, by Corollary $8, d\left(X_{t}\right) \leq 2$. On the other hand, $d\left(Y_{t}\right) \geq d\left(Y_{0}\right)=u$. 
Finally we note that the previous results don't remain true for pairs of groups in $\mathscr{L}_{\mathrm{ab}}$. Indeed, given $n \in \mathbb{N}$ there exist $X, Y \in \mathscr{L}_{\text {ab }}$ with $\operatorname{soc} X=\operatorname{soc} Y, \max \left(d\left(X_{0}\right), d\left(Y_{0}\right)\right) \leq$ 2 but $d\left(Y_{u n}\right)-d\left(X_{u n}\right)=u(n-1)$ for any positive integer $u$. Let $p$ be an odd prime and let $V$ be a vector space of dimension $n$ over the field $\operatorname{GF}(p)$. Moreover, let $H_{1}=\mathrm{GL}(n, p)$ and let $H_{2}$ be the subgroup of $\mathrm{GL}(n, p)$ generated by a Singer cycle of order $p^{n}-1$. Take the semidirect products $X=V H_{1}$ and $Y=V H_{2}$. Note that $X, Y \in \mathscr{L}_{\text {ab }}$ with $\operatorname{soc} X=\operatorname{soc} Y=V$ and that $d\left(X_{0}\right)=d\left(H_{1}\right)=2$, $d\left(Y_{0}\right)=d\left(H_{2}\right)=1$. Since $\operatorname{End}_{H_{1}} V=\mathrm{GF}(p)$ and $\operatorname{End}_{H_{2}} V=\mathrm{GF}\left(p^{n}\right)$, we have $r_{X}=n$ and $r_{Y}=1$. Moreover, $\mathrm{H}^{1}\left(H_{1}, V\right)=\mathrm{H}^{\mathrm{l}}\left(H_{2}, V\right)=0$ so $s_{X}=s_{Y}=0$. For any positive integer $u$, from Proposition 6 , we deduce $d\left(X_{n u}\right)=h_{X, n u}=1+u$, $d\left(Y_{n u}\right)=h_{Y, n u}=1+n u$.

\section{References}

[1] M. Aschbacher and R. Guralnick, 'Some applications of the first cohomology group', J. Algebra 90 (1984), 446-460.

[2] F. Dalla Volta and A. Lucchini, 'Finite groups that need more generators than any proper quotient', J. Austral. Math. Soc. Ser. A 64 (1998), 82-91.

[3] — - 'The smallest group with non zero presentation rank', J. Group Theory 2 (1999), 147-155.

[4] W. Gaschütz, 'Zu einem von B. H. und H. Neumann gestellten Problem', Math. Nachr. 14 (1955), 249-252.

[5] K. W. Gruenberg and K. W. Roggenkamp, 'Decomposition of the relation modules of a finite group', J. London Math. Soc. (2) 12 (1976), 262-266.

[6] W. Kantor and A. Lubotzky, 'The probability of generating a finite classical group', Geom. Dedicata $36(1990), 67-87$.

[7] A. Lucchini, 'On groups with $d$-generators subgroups of coprime index', Comm. Algebra 28 (2000), 1875-1880.

[8] A. Lucchini and F. Menegazzo, 'Generators for finite groups with a unique minimal normal subgroup', Rend. Sem. Mat. Univ. Padova 98 (1997), 173-191.

[9] A. Lucchini and F. Morini, 'On the probability of generating finite groups with a unique minimal normal subgroup', Pacific. J. Math. to appear.

[10] I. Pak, 'On the probability of generating a finite group', preprint, 2000.

Dipartimento di Matematica e Applicazioni

Università di Milano - Bicocca

Via Bicocca Degli Arcimboldi 8

20126 Milano

Italy
Dipartimento di Matematica

Università di Brescia

Via Valotti 9

25133 Brescia

Italy 
